\title{
Nuclear data for innovative fission reactors and fuel cycles
}

\author{
Jacques Bouchard
}

Advisor to the CEA General Administrator Chairman of the Gen IV International Forum

\begin{abstract}
The development of nuclear systems and technologies largely depends on the availability of nuclear data. Nuclear data thus represents input required to fulfil two sets of needs: those corresponding to generic issues, as well as those specifically adapted to the context.
\end{abstract}

Generic issues cover needs related to design and licensing purposes. Validation of models and codes used for simulation of operational, transient and accident conditions, increase of their accuracy through quantification and reduction of uncertainties, are achieved through confrontation with nuclear data, which contributes to greater optimization in conceptual design and licensing, thus impacting on the economics of the entire system.

However, in addition to these generic issues, specific needs stemming from the context shape the nuclear data required for the development of nuclear systems and technologies. For example, if we consider the present situation, it is characterized by signs of nuclear renaissance, driven by environmental concerns, scarcity of fossil fuels, soaring energy demand from developing countries, non proliferation concerns. As a result, new sets of criteria, requiring new sets of data, have to be accounted for, in setting up plans for new nuclear systems. Among these new criteria, those related for example to sustainability, which induce the necessity of closing the fuel cycle for, among other reasons, a better use of natural resources.

More generally, the present context clearly calls for the development of new reactor concepts. Predictions indicate an increase by a factor 2 of the world's energy needs, shifting from 10 to 20 GTOE in the period 2005-2050. To meet such an increase, nuclear energy is expected to play a more important role, with an increase of the world's nuclear capacity by a factor of about 4 over the same period (1400 GWe in 2050). However, during that timeframe, light water reactors using uranium fuel will continue to prevail, representing an installed capacity of $1200 \mathrm{GWe}$ in 2050 , versus $320 \mathrm{GWe}$ in 2005 . As a result, studies requiring data related to physics features are performed, to extend the burnup of these light water reactors. Their fuel burnup has been gradually increasing, driven by cost concerns. Current plans indicate that burnup might be increased in the [50-100] MWd/kg range. However, as burnup increases, the neutronic contributions of transuranics become predominant.

Other concerns, stemming from the need for enhanced safety, minimization of waste, preservation of resources, non proliferation issues, have lead to setting up an internationally

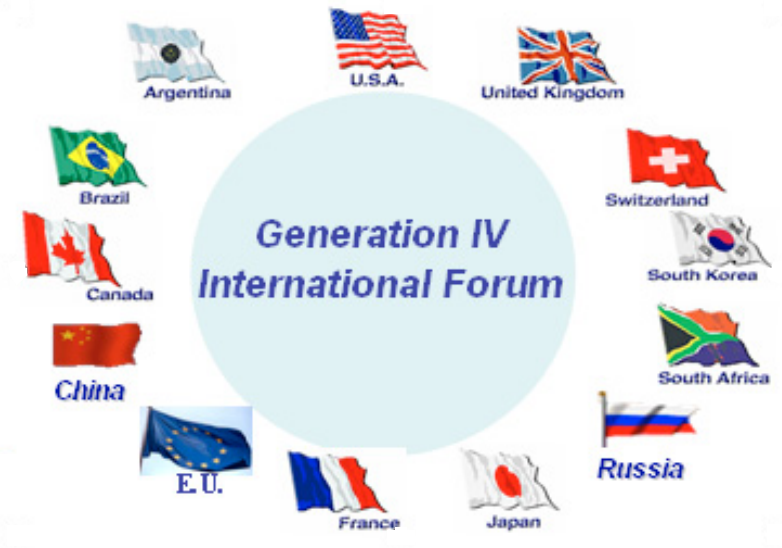

Fig. 1. The Generation IV International Forum.

shared R\&D, within the Generation IV Forum, expected to yield industrial results by 2030 .

For this next reactor generation and its associated fuel cycles, which rely on concepts involving numerous breakthroughs, nuclear data and computational methods will need to be reviewed, in order to guarantee computational results of very high standards.

The presence of a high content of transuranium elements in the fuel, required for waste minimization as well as multirecycling of plutonium, will call for extensive amount of data. So will the evaluations of power distributions (and their evolution with time) in the core and close to interfaces (for e.g., reflectors), as well as the study of reactivity effects due to innovative control rod systems and to innovative core architectures, aimed at drastically reducing the coolant void effect.

The use of non-standard materials (for, e.g., new fuel constituents and reflector materials) will call for specific validation.

Needs for potential cross section data will also arise. The characterization of irradiated fuel, including radiation emission characteristics, will allow for improved neutron cross 
sections for plutonium isotopes and minor actinides. Good insight will be required on fission capture (both in a wide energy range, including low energy resonances) and inelastic scattering cross sections.

Improved cross section data will be useful, especially on inelastic cross sections, for non conventional coolants (for, e.g., $\mathrm{Pb}, \mathrm{Bi}$ ), structural materials, and fuel matrix materials (e.g., $\mathrm{Zr}, \mathrm{Mg}, \mathrm{Ti}$ ).

As for fission product data, despite wide effort in the past, some improvement can be suggested through tighter target accuracy on reactivity loss during cycle.

Beyond neutron cross sections, the assessment of advanced nuclear systems requires improved data not only for neutron reaction data, but also for other nuclear parameters.

For decay and fission yield data, decay heat assessment will serve to assess high minor actinides fraction in the fuel.

For effective fraction of delayed neutrons, the presence in the fuel of a high content of minor actinides should be studied, as it lowers the effective delayed neutron fraction, making the reactor control more delicate.

Thermal scattering data should be collected, due to the changes in microstructure which can affect thermal scattering, and thus have an impact on safety and performance parameters.

As for gamma production data, they are of relevance for power distribution assessment, in particular at interfaces of innovative burner reactors. Improved evaluation and possibly experiments are still required.

The role of nuclear data for future systems should be carefully assessed. The impact of cross section uncertainties has to be quantified in order to define priorities and strategies for improvement. Work is underway under the supervision of the NEA-Nuclear Science Committee. Results are available for reactor and fuel cycle related data for most Gen IV systems, as well as for GNEP's ARR, LWRs with extended burn up, and Accelerator Driven Systems. This work has been achieved through strong international effort, recently started with the aim of producing reliable, science-based, varianceco-variance data.

If we consider, for example, Gen IV fast reactors, breeders or burners, fuels, structures and reflectors might contain new materials $(\mathrm{Zr}, \mathrm{Si})$. Uncertainties can reach high levels: on keff and reactivity loss/cycle, they can reach up to $2 \%$ and $1 \%$ $\Delta \mathrm{k} / \mathrm{k}$. On void reactivity coefficient and Doppler reactivity coefficient, they can reach $20 \%$ and 5 to $10 \%$. These uncertainties often turn out to be larger than target accuracies.

In order to best collect relevant and reliable data, strong cooperation is required between nuclear physicists, reactor physicists and reactor system designers, at an international level. Moreover, in view of the high accuracy requirements related to both safety and economic goals, innovative experiments, experimental techniques and theoretical developments are required, which would allow for the elaboration of sophisticated simulation on "basic principles". Also, "Nuclear Physics" differential experiments and "Integral" (i.e., reactor physics oriented) experiments should play a complementary

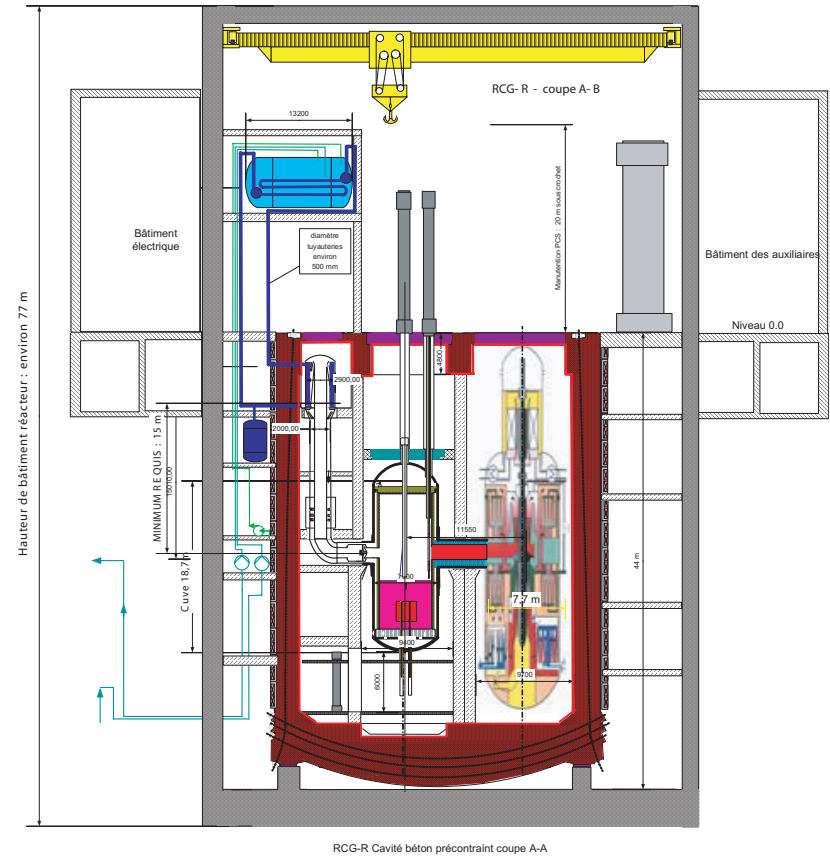

Fig. 2. An example of Gen IV fast reactor.

role, and should be well understood by both communities. Unfortunately, at this point, very few facilities are available.

As Al Henry [1] puts:

"Good luck is not a lasting substitute for good science. A computer program should be evaluated on the basis of the nuclear data and reactor theory that underlie it. It is ironic that many of those who claim that our state of knowledge is such that efforts to develop more accurate methods of analysis can be reduced and that clean, unambiguous experiments are no longer necessary really don't believe the predictions of the theoretical models in present use. If it can possibly be managed financially, they always seem to want a full mock-up of any proposed design before making a decision to accept it. My conviction is that it is both uneconomical and unnecessary to allow this situation to persist".

A possible flow chart to achieving advanced reactor codes can thus be as follows:

Existing theory, coupled to differential measurements and integral experiments can be used to build up nuclear data files with covariances. In turn, these files help make up group libraries with covariances, which, combined to data and uncertainties for other disciplines, will lead to advanced reactor codes, and finally, to nominal reactor parameters and uncertainties.

A large number of "clean" integral experiments have been performed worldwide in the past, and have been collected and compiled at OECD/NEA, within the framework of the Nuclear Science Committee [2]. Selected new integral experiments should be carefully defined to meet well specified objectives and performed within the frame of large international collaborations. For example, the CEA-Cadarache MASURCA facility is presently being refurbished to support innovative future fast reactor programs. 

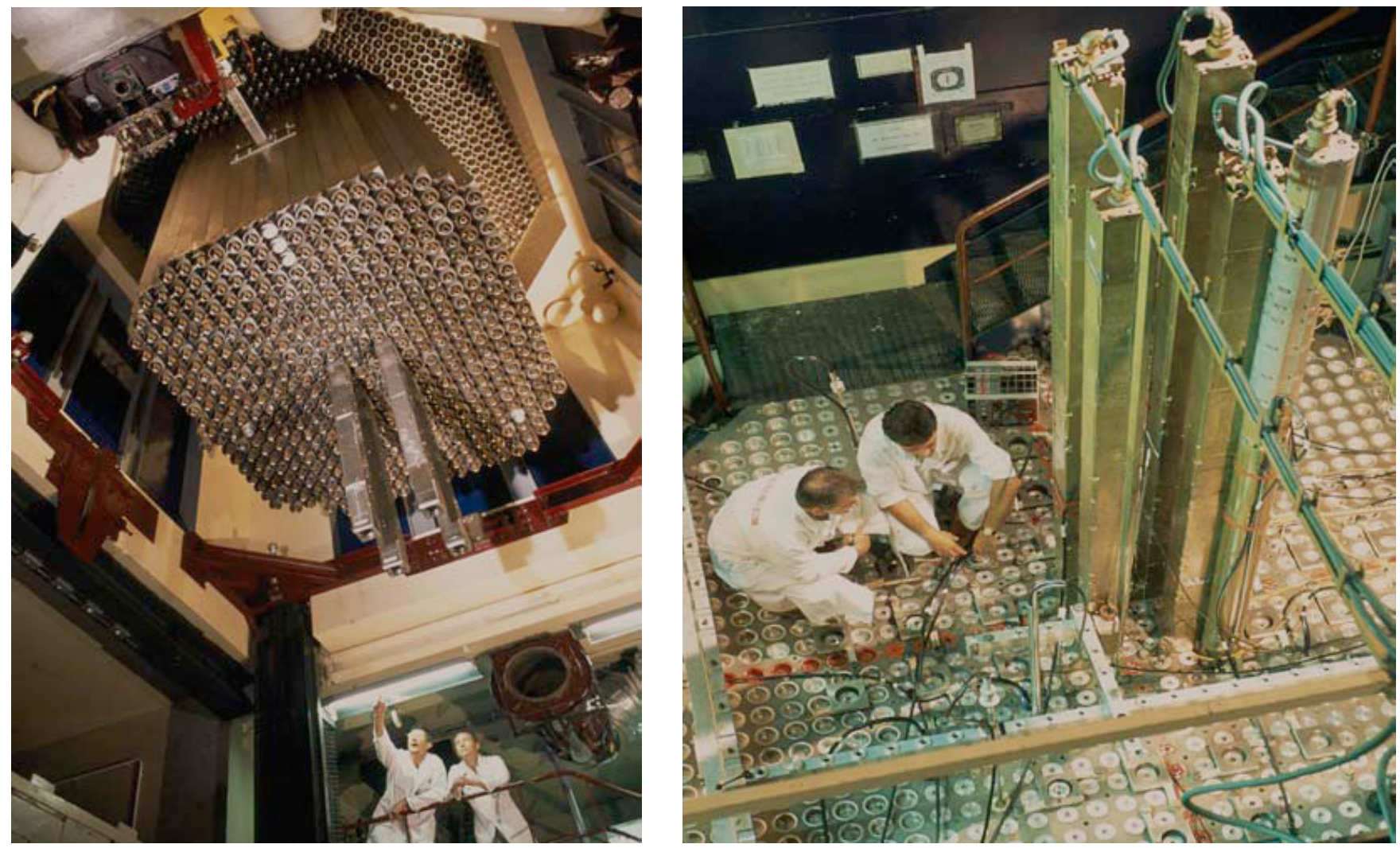

Fig. 3. The CEA-Cadarache MASURCA facility.

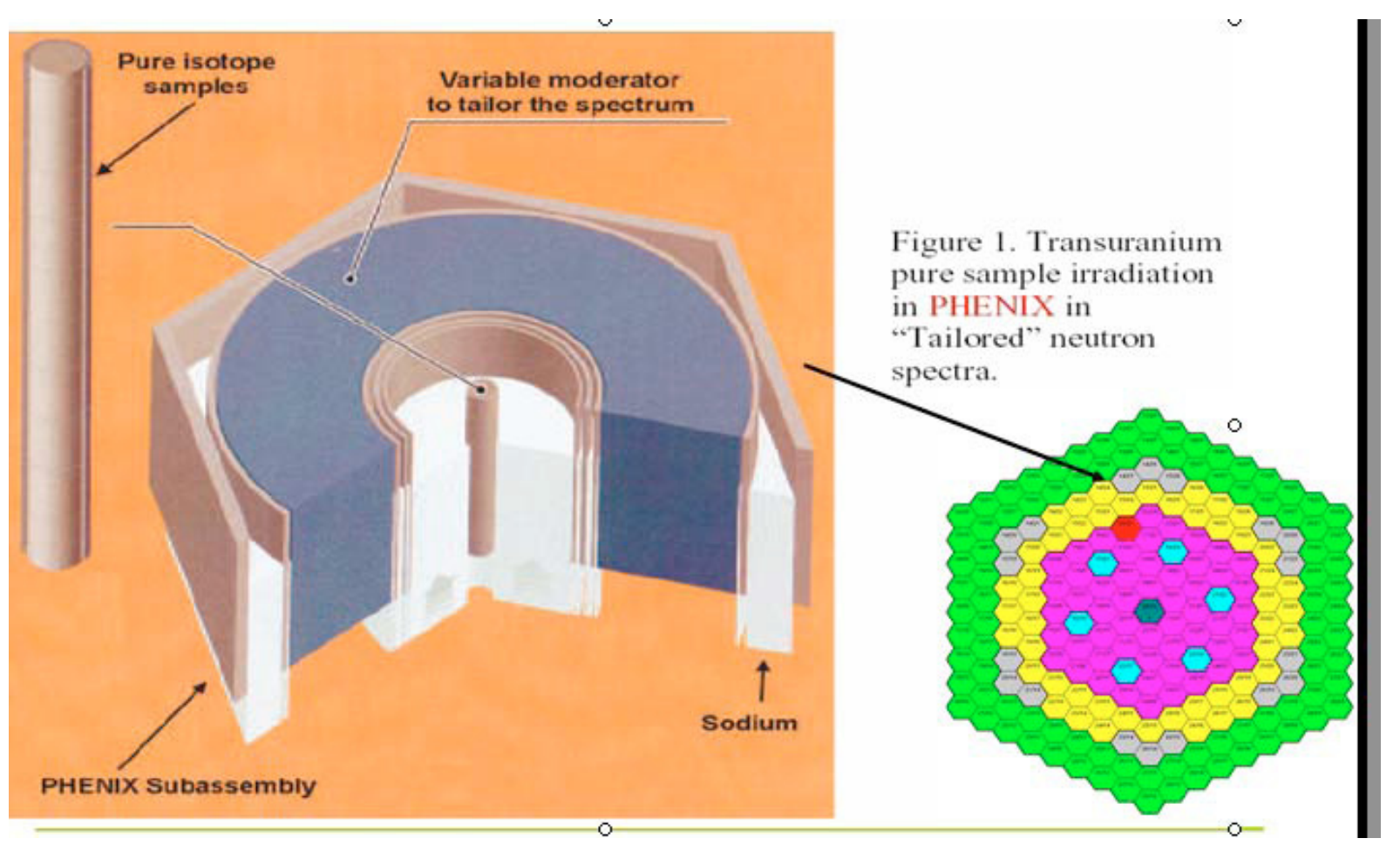

Fig. 4. Transuranium pure sample irradiation in Phenix in "Tailored" neutron spectra. 
Also, innovative irradiation experiments are being carried out (for example, transuranium pure sample irradiation in Phenix in "tailor" neutron spectra).

In conclusion, it has to be stated that nuclear data still have a key role to play in future energy supply. Specific nuclear data needs have already been identified for existing as well as for new concepts. They undoubtedly will have an impact on the assessment of new concepts, on safety features and economics. Covariance data are becoming available, and will help shift towards more efficient R\&D models. Experimental capabilities and expertise should be kept alive, as they are of upmost importance for the sustainability of Nuclear Energy.

\section{References}

1. A.F. Henry, Nuclear Reactor Analysis (The MIT Press, Cambridge, MA, May 15, 1975).

2. International Handbook of Evaluated Reactor Physics Bench mark Experiments, NSCDOC-2006-1, March 2006 Edition OECD NEA. 